\title{
The Robustness of Urban Rail Transit Network based on Complex Network Theory
}

\author{
Yiran $\mathrm{Gu}^{1, a}$, Cheng $\mathrm{Li}^{2, \mathrm{~b}}$ \\ ${ }^{1}$ Department of Electrical Engineering and Automation, Nanjing University of Posts and \\ Telecommunications, Nanjing, 210023, China \\ ${ }^{2}$ Department of Electrical Engineering and Automation, Nanjing University of Posts and \\ Telecommunications, Nanjing, 210023, China
}

aemail: guyr@njupt.edu.cn, bemail:licheng010561@163.com

Keywords: Rail Transit Network; Random Attack; Malicious Attack; Robustness

\begin{abstract}
The research object is based on public rail transit network of two cities in China. The robustness analysis is carried out on the rail transit network which is constructed by the transfer stations. On the basis of the existing robustness evaluation indexes, we put forward alpha index and isolated node ratio as invulnerability measure of the rail transit network and reviewed the invulnerability of rail transit network under two attack patterns. The simulation results show that the empirical object has the robustness under random attack and vulnerability under malicious attack.
\end{abstract}

\section{Introduction}

With the speeding up of urbanization and people's improving requirements of traffic efficiency in our country, the demand of the construction of urban rail transit is increasingly significant. Rail transit will enter a relatively perfect network construction and operation stages. People also put forward higher requirements for the stability showed by urban rail transit network in the case of internal fault and external attack.

The robustness of urban rail transit network refers to the ability of traffic network system to provide alternative routes in the case of accident or interference. The robustness analysis of rail transit network can be instructional suggestions on construction of rail transit. As a result, it can improve the quality of rail transit operation and give full play to the advantages of rail transit.

The robustness of complex network has also been a research focus of domestic and foreign scholars [1] [2]. Albert [3] etc. compared the connectivity of random networks and scale-free networks and concluded that the two networks under random attack and malicious attack have different robustness. Holme P [4] presented the global efficiency and the relative size of the biggest connected sub-graph to measure network performance. Cohen [5] etc. analyzed the robustness of the Internet under the condition of random attack and malicious attack by using the percolation theory. Yongchao Jiang [6] made a reliability analysis for Chinese railway network model by using global efficiency of the network.

This paper puts forward alpha index and isolated node ratio as two parameters on the basis of robustness evaluation index such as global efficiency, average path length, relative size of the biggest connected sub-graph. In this paper we analyze the network robustness respectively in the line replacement capacity and the overall destruction of the network. In the last, we made empirical analysis for Nanjing and Beijing rail transit network in 2020.

\section{The Construction of Rail Transit Network Model}

$G=(V, E)$ refers to an undirected and un-weighted network, and $V$ represents a collection of network nodes, and $E$ represents a collection of network edges. Each edge is connected with two nodes and a pair of nodes is connected by one edge at most. $n$ represents the number of nodes in the network that is $n=|v|$, and $e$ represents the number of edges that is $e=|E|$. In order to facilitate the research, this paper makes the following assumptions in the construction of the rail 
transit network.

(1) This paper will focus on the transfer stations and the terminal stations of rail transit network, and ignore the common sites, and do not consider the connection weights between the network nodes.

(2) The rail transit network is defined in the space P.

(3) Each attack only destroys a site in the topology, and the edges of the site are removed after the site has been destroyed.

This article selected data of Beijing and Nanjing rail transit network in 2020. Nanjing rail transit network contains 79 nodes and 130 edges, and Beijing rail transit network includes 82 nodes and 133 edges. The topological structure is shown below.

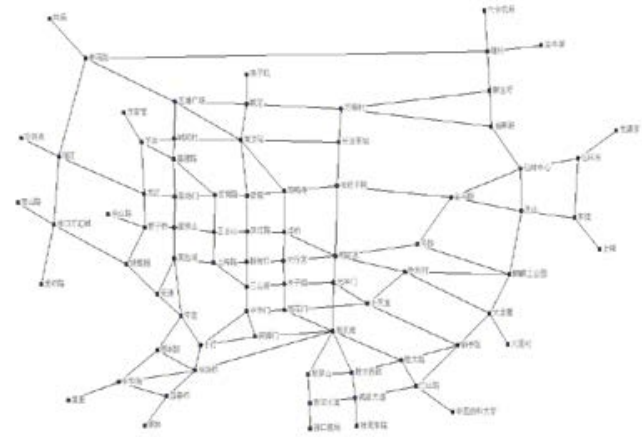

Fig.1 Nanjing rail transit network

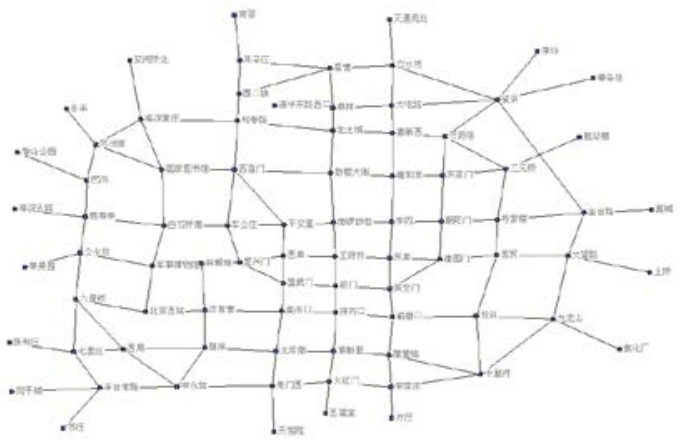

Fig.2 Beijing rail transit network

\section{Network Attack Strategy}

In this paper, we consider random attack and malicious attack as two kinds of node deletion strategies. Random attack indicates that the network node is randomly deleted, and the malicious attack says that the network node is destroyed according to a certain strategy. In view of the research object is the rail transit network, node betweenness [10] better reflect the transmission of nodes in the network. The higher nodes betweenness it is, the greater importance of the node. The malicious attack strategy shows that the node is attacked by node betweenesse from big to small. The node betweeeness is defined as follows.

$$
B C_{i}=\sum_{s \neq i \neq t} \frac{n^{i} s t}{g_{s t}}
$$

In this formula, $g_{s t}$ is the number of the shortest path from node $s$ to node $t, n_{s t}^{i}$ is the number of shortest path from node $s$ to node $t$ through the node $i$.

\section{Classic Parameters}

\subsection{Global Efficiency}

The global efficiency of the network [10] can be calculated by the shortest distance between nodes. Calculating formula is written as

$$
E_{\text {global }}=\frac{1}{n(n-1)} \sum_{i \geq j} \frac{1}{d_{i j}}
$$

The $d_{i j}$ is the distance between the node $i$ and the node $j$, which is the number of edges in the shortest path that connects two nodes, and $n$ is the number of nodes in a network. Global efficiency represents the overall connectivity of urban rail transit network, $0 \leq E_{\text {global }} \leq 1$. When $E_{\text {global }}=1$ indicates that any two nodes are connected in the network, and the overall connectivity and robustness of the network is the highest. When $E_{\text {global }}=0$ says that the nodes in the network are not connected with each other, corresponding to the minimum of the network robustness. 


\subsection{Average Path Length}

Average path length [10] of the network is defined as the average distance between any two nodes. Calculating formula is written as follows.

$$
L=\frac{1}{\frac{1}{2} n(n-1)} \sum_{i \geq j} d_{i j}
$$

The average path length describes the average number of transfers between any two nodes. Albert [3] etc. studied the robustness of Internet, and found that the average path length becomes larger firstly then smaller with the increase of the proportion of removal nodes. They defined the proportion $f_{c}$ of removal nodes that caused the average path length of the network to achieve the peak value is robust index. The greater $f_{c}$, the better the network robustness.

\subsection{Relative Size of the Biggest Connected Sub-graph}

The biggest connected sub-graph of the network indicates that the sub-graphs that all nodes in the graph are connected by the least edges .The relative size of the biggest connected sub-graph [10] is the ratio of the number of nodes in the biggest connected sub-graph and the number of nodes in network. Calculating formula is written as follows.

$$
S=\frac{N^{\prime}}{N}
$$

$N^{\prime}$ refers to the number of nodes in the biggest connected sub-graph after external attack or internal fault . $N$ refers to the total number of network nodes that have not been attacked.

\section{New Evaluation Index}

\subsection{Alpha Index}

The ratio of number of the actual loops and the maximum number of loops which may exist in the network, is called alpha index. The calculating formula is shown as follows.

$$
\alpha=\frac{e-n+1}{2 n-5}
$$

$e-n+1$ is the number of basic loops in the network. It represents the difference between the number of network edges and the number of its spanning tree edges. The maximum number of edges in a planar network [8] is $3 n-6$, and the maximum number of basic loops in the network of $n$ nodes is $2 n-5$. Alpha index is a measure of the loop performance in the network and the range of value change is $0 \sim 1$.

\subsection{Isolated Node Ratio}

In the process of random attack and malicious attack, isolated nodes can not be connected to the whole network and the significance of the existence of nodes is lost. To some extent, the more the number of isolated nodes is, the worse connectivity of the network. In this paper, the isolated nodes ratio describes the damage degree of the whole network under the two attack patterns. We use $P$ to represent the isolated node ratio. 


\section{Simulation and Analysis}

\subsection{The Simulation Analysis of Nanjing Rail Transit Network Robustness}

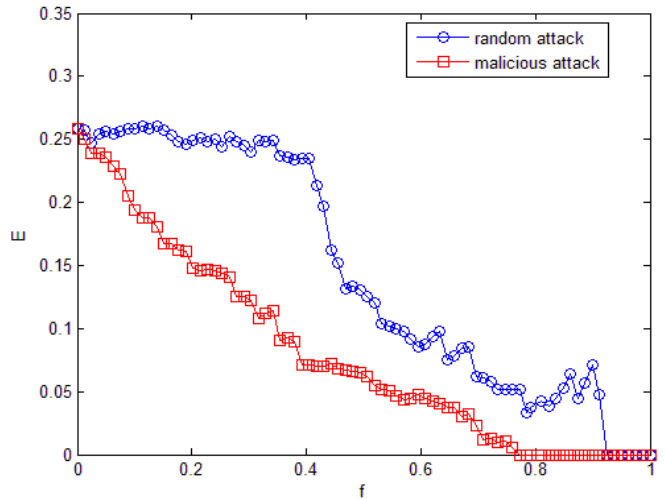

(a) Global efficiency

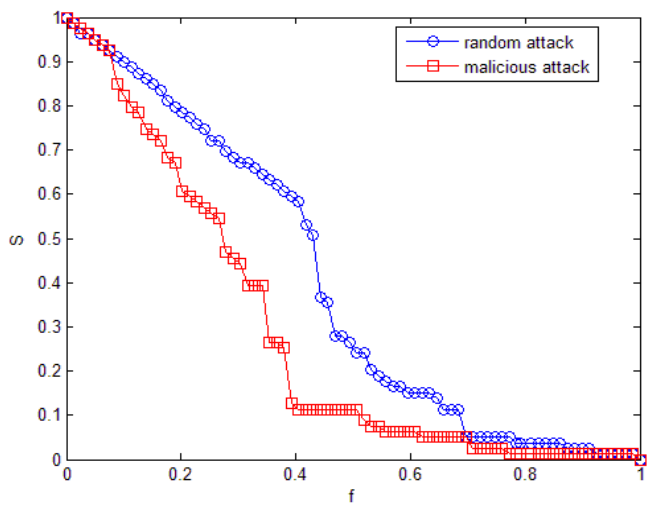

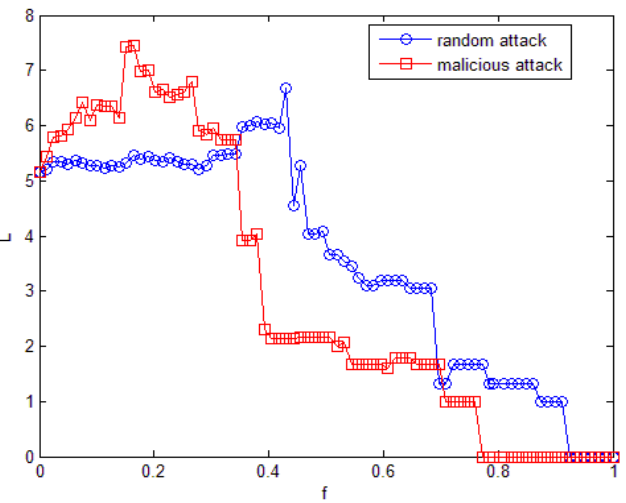

(b) Average path length

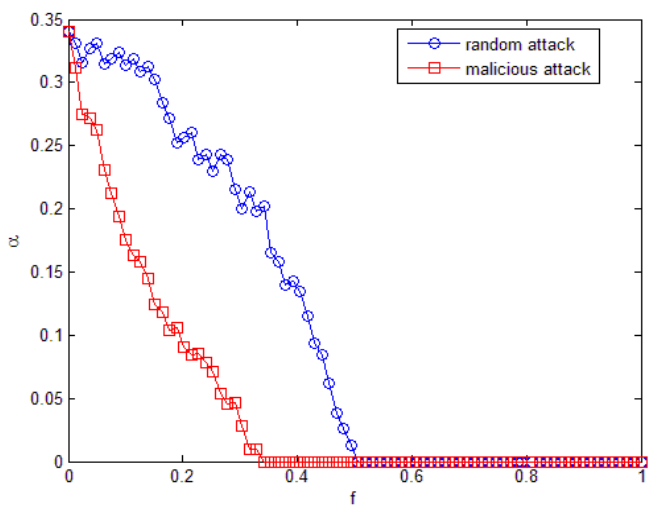

(d) Alpha index

(c) Relative size of the biggest connected sub-graph

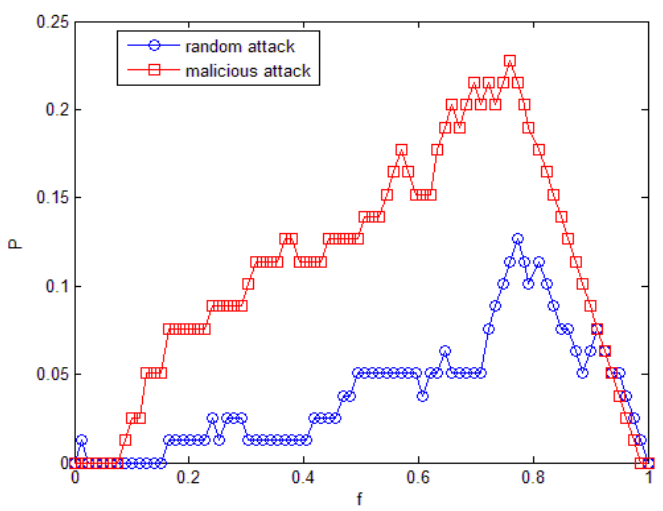

(e) Isolated node ratio

Fig.3 Parameter variation under two attack modes (Nanjing) 


\subsection{The Simulation Analysis of Beijing Rail Transit Network Robustness}

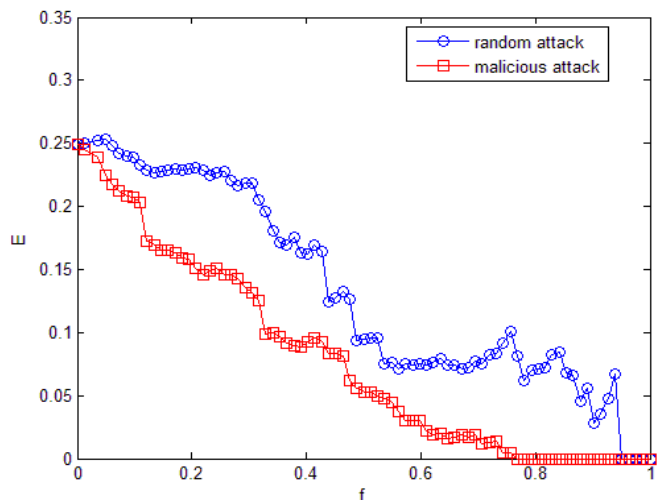

(f) Global efficiency

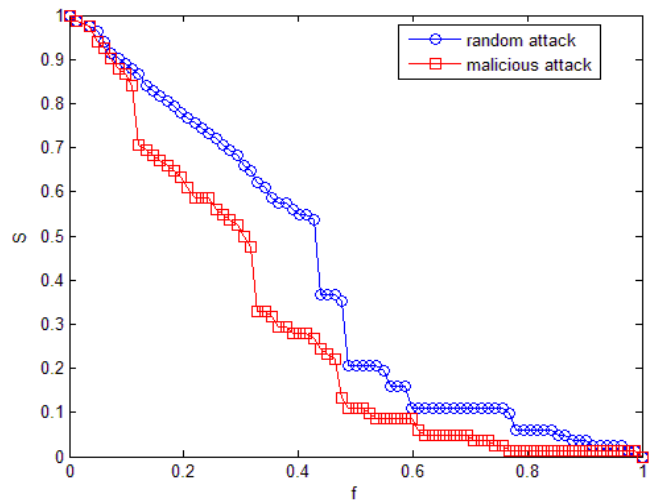

(h) Relative size of the biggest connected sub-graph

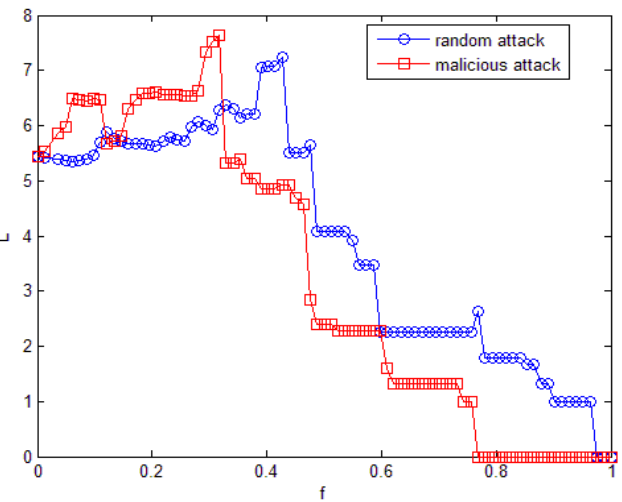

(g) Average path length

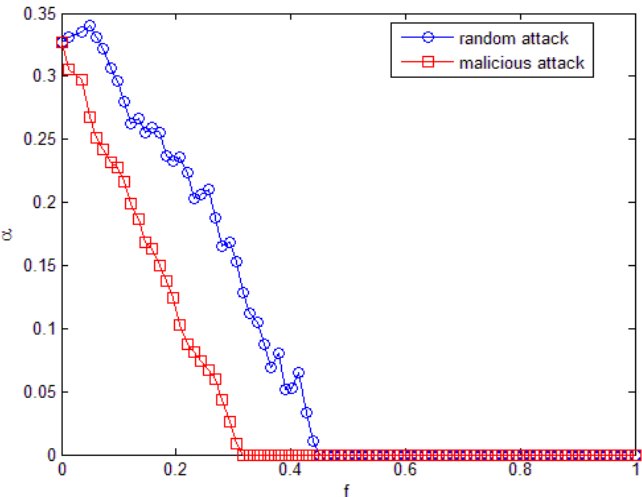

(i) Alpha index

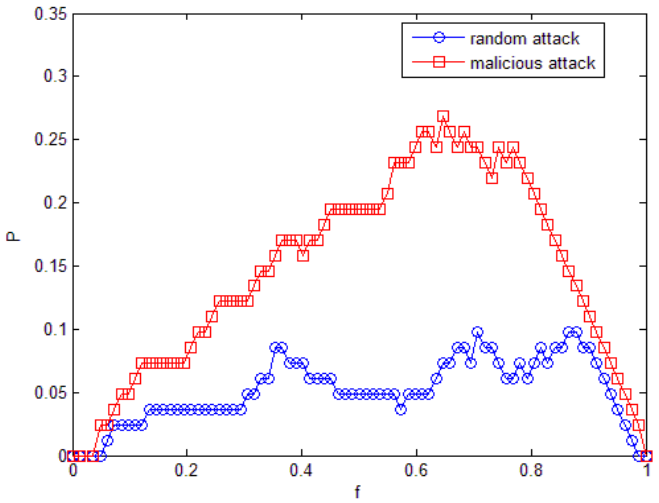

(j)Isolated node ratio

Fig.4 Parameter variation under two attack modes (Beijing)

\subsection{Analysis}

From Fig.3 (a), we can see that global efficiency trend of Nanjing rail transit network under the two attack modes. In the proportion of $[0,0.9]$, the global efficiency of the network under malicious attack is always lower than that of the random attack. From Fig.4 (f), we can know that Beijing rail transit network also reflects the robustness under random attack and the vulnerability under malicious attack.

Fig.3 (b) illustrates that the average path length of Nanjing rail transit network is first become large and then become smaller with the increase of removal nodes proportion, it also shows that random attack has $f_{c}=0.43$ and malicious attack has $f_{c}=0.16$. For the Beijing rail transit network, the Fig.4 (g) indicates that random attack has $f_{c}=0.42$ and malicious attack has $f_{c}=0.32$.

The change trend of the relative size of the biggest connected sub-graph with the nodes removal 
gradually becomes smaller. In the node deletion proportion [0, 0.8], the relative size of the biggest connected sub-graph under the malicious attack is always lower than that of the random attack.

The alpha index describes the line replaceable capacity when it is attacked. For the rail transit network in Nanjing and Beijing, when the proportion of random attack nodes reaches 0.5 and 0.42 , respectively, and the value of alpha index is 0 . However, the proportion of the malicious attack node reaches 0.34 and 0.3 , the value of alpha index runs up to 0 . It indicates that the network must be a non-connected network.

Fig.3 (e) and Fig.4 (j) indicate that the isolated node ratio trend firstly becomes large and finally close to zero. For Nanjing rail transit network, when $f=0.76$ isolated node ratio achieves the highest value under two modes, and the highest value under random attack is 0.11 , and the maximum value is 0.23 under malicious attack. For Beijing rail transit network, the highest value of isolated node ratio is 0.09 under random attack, and the maximum value is 0.26 under the malicious attack. With the isolated nodes being attacked, isolated node ratio eventually trend to zero.

Table 1 Time complexity of robustness evaluation indexes

\begin{tabular}{cccccc}
\hline $\begin{array}{c}\text { Evaluation } \\
\text { parameters }\end{array}$ & $\begin{array}{c}\text { Global } \\
\text { efficiency }\end{array}$ & $\begin{array}{c}\text { Average } \\
\text { path length }\end{array}$ & $\begin{array}{c}\text { The relative size of the } \\
\text { biggest connected } \\
\text { sub-graph }\end{array}$ & $\begin{array}{c}\text { Alpha } \\
\text { index }\end{array}$ & $\begin{array}{c}\text { Isolated node } \\
\text { ratio }\end{array}$ \\
\hline $\begin{array}{c}\text { Time } \\
\text { complexity }\end{array}$ & $o(m n)$ & $o(m n)$ & $o(1)$ & $o(1)$ & $o(1)$
\end{tabular}

The results indicate that transit network in Beijing and Nanjing is robust against random attack and vulnerable to malicious attack. It is reasonable to use the alpha index and the ratio of isolated nodes as the robustness evaluation parameters.

\section{Conclusion}

Based on complex network theory, this paper makes an empirical analysis on the robustness of Nanjing and Beijing rail transit network, and adopts random attack and malicious attack as two strategies. Based on the global efficiency of network, the relative size of the biggest connected sub-graph, the average path length, we propose alpha index and the isolated node ratio to evaluate network robustness. From line replacement capacity and overall damage of the whole network to measure the invulnerability of the network and the simulation analysis is carried out. By new parameters we can draw a conclusion that Beijing and Nanjing rail transit network have robustness under random attack and vulnerability under malicious attack. The research scope of applying complex network theory to the analysis of urban traffic network is very wide. This study is only a point, and the next step is to join the temporal variation of the rail transit network to analyze the robustness. It will be significant to the construction and stable operation of urban rail transit network.

\section{Acknowledgement}

In this paper, the research was sponsored by the Foundation of the Ministry of Education of China for the Humanities and Social Sciences Research Program (Project No. 15YJZH016) .

\section{References}

[1]Bollobas B, Riordan O. Robustness and Vulnerability of Scale-free Random Graphs [J] .Internet Math, 2003 ,1:1-35

[2]Jie Gao , Qizhou Shi. Definition and Evaluation Modeling of Metro Network Invulnerability [J].Journal of the China Railway Society .2007, 29 (3):29-30. 
[3]Albert R, Jeong H, Barabasi A L. Error and Attack Tolerance of Complex Networks [J]. Nature, 2000, 406: 378-382.

[4]Holme P, Kim B J. Attack Vulnerability of Complex Networks [J].Phys Rev E, 2002, 65(6); 066109.

[5]Cohen R, Erez K, ben-Avraham D. Resilience of the Internet to Random Breakdowns [J]. Phys Rev Lett , 2000, 85(21): 4626-4628.

[6] Yongchao Jiang. Reliability Analysis of Railway Network Based on Complex Network Theory [D].Chengdu:Southwest Jiao Tong University,2011.

[7]Chunquan He . Research on Robustness during Network Evolution [D].Shanghai Jiao Tong University,2009

[8]Huiquan Sun. Graph Theory and Its Applications [M], Beijing: Science Publishing Company,2004

[9] Shi Dinghua. Degree Distribution Theory of Network [M],Beijing:Higher Education Press,2011

[10]Xiaofan Wang ,Xiang Li , Guanrong Chen,Introduction to network science[M],Beijing:Higher Education Press,2012

[11]Bin Wang, Application Research of Small-world Network Theories in Traffic Network [D],Nanjing: Nanjing University of Aeronautics and Astronautics,2013

[12]Yihong $\mathrm{Hu}$.Complexity Research of Traffic Network Based on Complex Network [D].Shanghai: Fudan University,2008 